\title{
Experimentelle Untersuchung der Dauerhaftigkeit von Tunneltüren für schnellbefahrene Bahntunnel der ÖBB
}

\author{
DI Dr. Helmut Steiner, ÖBB Infra PNA PLK 1, Graz \\ DI Dr. Michael Reiterer, REVOTEC zt gmbh, Wien
}

\section{Kurzfassung}

Bahntunnels stellen u.a. hinsichtlich Ausstattung, Ausrüstung, aerodynamischen Belastungen bei Zugfahrten sehr spezielle Bauwerke dar, bei denen die unterschiedlichsten Randbedingungen und teilweise auch widersprüchliche Anforderungen zu erfüllen sind. Eine von vielen Kernkomponenten sind Tunneltüren, die wiederum einen zentralen Bestandteil des Sicherheitskonzeptes repräsentieren, indem sie den sicheren Bereich vom Ereignis trennen und daher eine entsprechend widerstandsfähige Abschottung auch bei Brandereignissen darstellen müssen.

In dieser Hinsicht ist es von großer Bedeutung Tunneltüren einzubauen, die diesen Anforderungen entsprechen und auch hinsichtlich Wartung und Instandhaltung bzw. Lebenserwartung die von Seiten der ÖBB an sie gestellten Erwartungen erfüllen.

Für das Neubauprojekt der Koralmbahn Graz - Klagenfurt wird nun versucht NEUE Tunneltüren zu definieren, zu prüfen und schlussendlich zu beauftragen bzw. einzubauen, die diesen Erwartungen entsprechen und somit eine neue ÖBB-Tunneltüren-Evolutionsstufe repräsentieren sollten.

\section{Abstract}

A Railway tunnel represent in relation of the tunnel equipment and the aerodynamic loads during train journeys a very special structure in which the most varied of boundary conditions and sometimes also contradicting requirements have to be met.

One of the many core components are tunnel doors, which in turn represent a central component of the security concept by separating the safe area from the incident and therefore have to represent a correspondingly resistant barrier even in the event of fire.

In this regard, it is of great importance to install tunnel doors that meet these requirements and also meet the expectations placed on them by the ÖBB in terms of servicing and maintenance or life expectancy.

Vortrag STEINER / REITERER

Dynamik Tage Wien 2021 
For the new construction project Koralmbahn Graz - Klagenfurt, attempts are now being made to define, test and finally commission and install NEW tunnel doors that meet these expectation and should represent a new evolutionary stage of ÖBB tunnel doors.

\section{Einleitung}

Eine moderne und effiziente Verkehrsinfrastruktur ist eine wesentliche Grundvoraussetzung für eine positive wirtschaftliche Entwicklung von Regionen, Ländern und Staaten. Auf Grund der ökologischen Vorteile fällt dem System Eisenbahn dabei eine besondere Rolle zu. Im mitteleuropäischen Zentralraum führen die topografischen Verhältnisse, bedingt durch den trennenden Alpenbogen, bei der Umsetzung einer modernen Eisenbahninfrastruktur häufig zu sehr langen Tunnelobjekten. Um einen qualitativ hochwertigen, verfügbaren und sicheren Eisenbahnbetrieb zu gewährleisten, sind eine Vielzahl von technischen Einbauten erforderlich, welche aus einem reinen Ingenieurbauwerk im Rohbau erst die eigentliche "Hightech-Anlage" für einen zukunftweisenden Bahnbetrieb macht.

Die technische Ausstattung/Ausrüstung eines Bahntunnels ist ein Balanceakt zwischen den unterschiedlichsten und teilweise konträren Anforderungen. Oberstes Ziel muss es aber sein, Komponenten zur Anwendung zu bringen, die eine hohe Zuverlässigkeit, geringe Ausfallwahrscheinlichkeit und lange Wartungszyklen haben.

Die Koralmbahn (KAB) als Teil des rd. 1.800 km langen Baltisch-Adriatischen Korridors im TEN-Kernnetz zählt zu den bedeutendsten Verkehrsinfrastrukturprojekten in Europa bzw. Österreich. Diese zweigleisige, elektrifizierte Neubaustrecke verbindet auf einer Länge von rund $130 \mathrm{~km}$ die Städte Graz und Klagenfurt und stellt mit dem Koralmtunnel (KAT) die östlichste große Alpenquerung dar [5; 6; 11].

Dieses Kernstück besitzt eine Länge von 32,9 km und eine maximale Überlagerung von rd. $1.200 \mathrm{~m}$. Er zählt zu den längsten Eisenbahntunneln der Welt und besteht aus zwei einspurigen Fahrtunnel mit einem lichten Tunnelnettoquerschnitt im ausgebauten Zustand von rd. $42 \mathrm{~m}^{2}$. Alle $500 \mathrm{~m}$ sind die beiden Fahrtunnel mit Querschlägen verbunden und in der Tunnelmitte befindet sich eine Nothaltestelle mit einer Länge von rd. $900 \mathrm{~m}$.

\section{Vortrag STEINER / REITERER}

Dynamik Tage Wien 2021 
- $\mathrm{KAB} / 127 \mathrm{~km}$ zweigleisig elektrifiziert

- KAT/ 32,8 km - zweiröhrig eingleisig

- 2 neue IC Bf. + 10 weitere Bf.

- $250 \mathrm{~km} / \mathrm{h}$ Höchstgeschwingkeit

- Flachlandbahn<1\%

- 45 min. Graz - Klagenfurt

- 2,5 Std. Wien - Klagenfurt
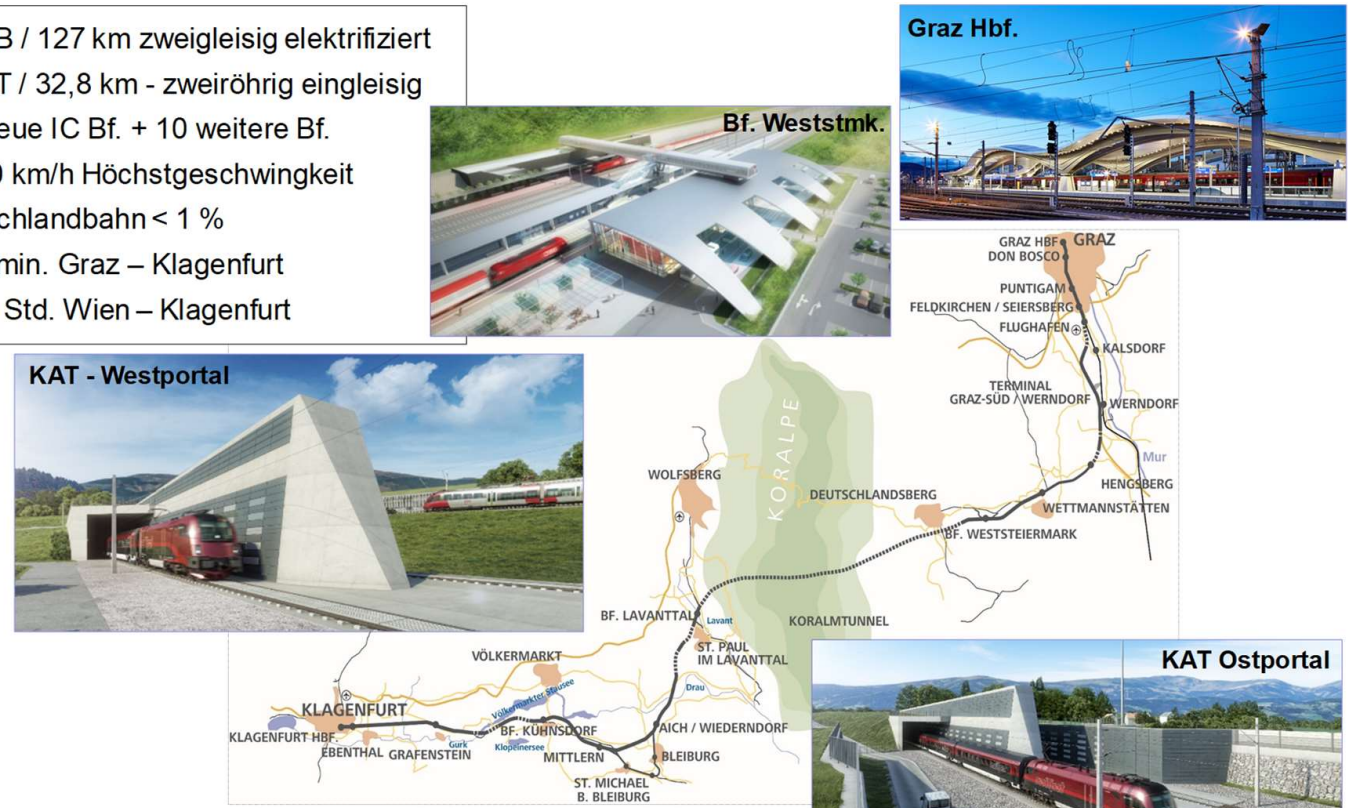

FUGHAFEN

KALSDORF

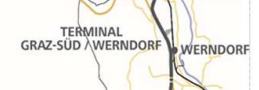

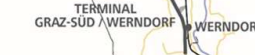
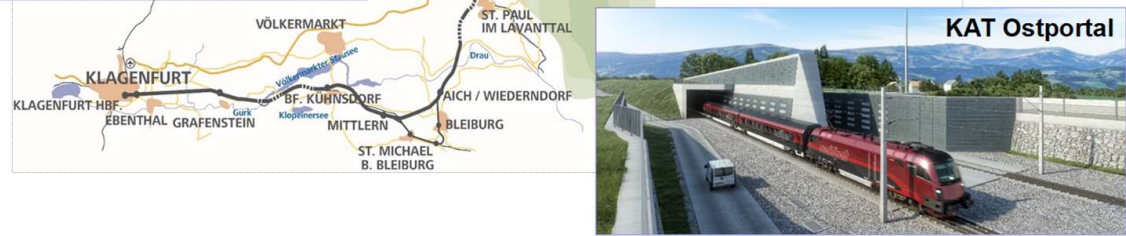

Abb. 1: Übersichtsdarstellung der Koralmbahn / KAB Graz - Klagenfurt [ÖBB]

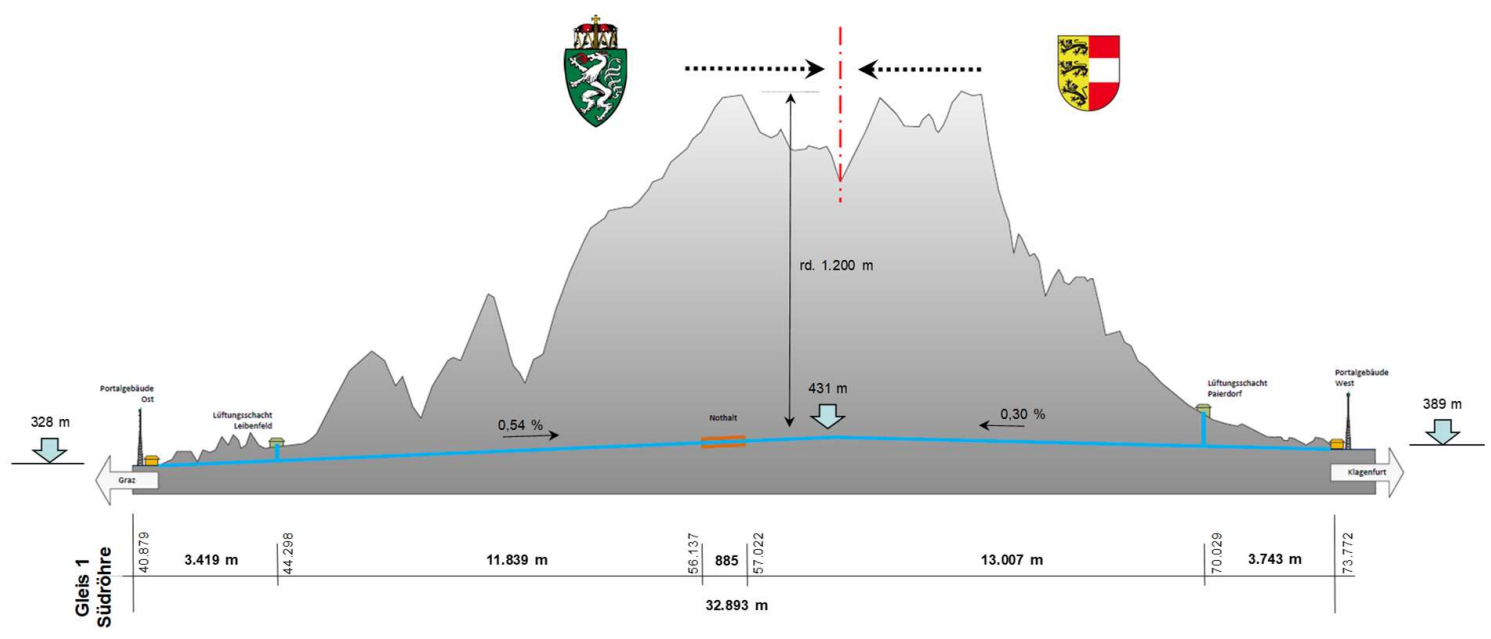

Abb. 2: Übersichtslängenschnitt des Koralmtunnels / KAT [ÖBB]

Die aktuellen Arbeiten befinden sich im Übergang vom Rohbau zur Bahntechnischen Ausstattung (Feste Fahrbahn, Oberleitung, Energieversorgung, Telekom-/ u. maschinelle Anlagen, wie auch Tunneltüren). Eine Inbetriebnahme des gesamten Systems ist mit Sommer 2025 für Güterzugs- und für Ende 2025 für Personenverkehre vorgesehen.

\section{Vortrag STEINER / REITERER}

Dynamik Tage Wien 2021 


\section{Aerodynamische Druck-/ Sogbelastungen in Bahntunnels}

Eine große Herausforderung stellt die ermüdungssichere Bemessung der unterschiedlichen technischen Einbauten im Fahrtunnel dar. Bei Zugfahrten kommt es zu äußerst komplexen Druck-/ und Sogwirkungen, die sich in ihrer physikalischen Wirkungsweise in druckwellen- und in strömungsinduzierte Belastungen unterteilen lassen.

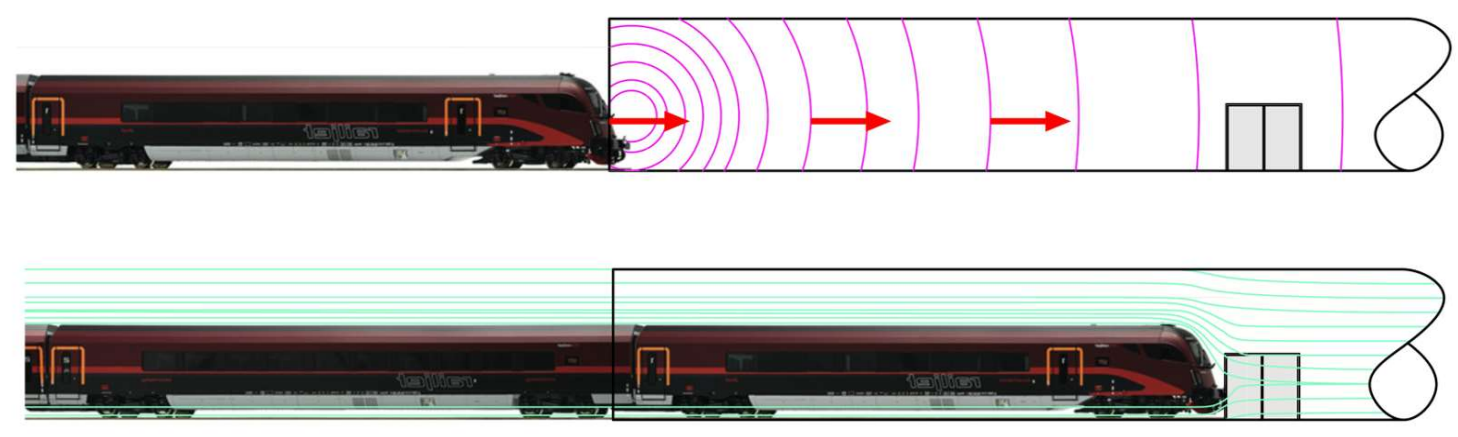

Abb. 3: Systemdarstellung von druckwellen- und strömungsinduzierten Belastungen [Revotec]

Die druckwelleninduzierten Belastungen entstehen bei der Ein- und Ausfahrt des Zuges in den Tunnel und sie wirken auf druckdicht abgeschlossene Bauteile wie z. B. Tunneltüren. Sie erreichen im Fall des KAT bei Zuggeschwindigkeiten mit bis zu 250 km/h eine Größe von rd. $+10,5 \mathrm{kN} / \mathrm{m}^{2}$ und - 12,0 kN/m². Die strömungsinduzierten aerodynamischen Belastungen entstehen bei der direkten Vorbeifahrt des Zuges am Ort des jeweiligen Bauteils im Tunnel und sie wirken auf umströmbare Bauteile wie z. B. Signalschilder. Diese Belastungen erreichen im Fall des KAT eine Größe von rd. +/- 4,1 kN/m².

Am Betrachtungsort des jeweiligen Einbauteils im Tunnel kommt es weiters zu mehrfachen Belastungseinwirkungen (Lastzyklen), die sich durch Reflexionen der Druckwellen am gegenüberliegenden Tunnelportal oder bei signifikanten Querschnittsänderungen bzw. Tunnelverzweigungen ergeben. Diese mehrfachen Belastungswirkungen führen zu einer Ermüdungsbelastung der Einbauten im Tunnel. In der Bemessung ist im Fall KAT eine Lastgröße von +/- 7,5 kN/m² mit 1 Millionen Zyklen aufzubringen.

\section{Vortrag STEINER / REITERER}

Dynamik Tage Wien 2021 


\section{Türen / Toren - allgemein}

Türen lassen ein, sperren jedoch auch aus. Sie schaffen Sicherheit, Geborgenheit, Schutz gegen das Wetter, gegen wilde Tiere, ungebetene Gäste und drohende Feinde. Türen gibt es seit Menschen Unterkünfte von unterschiedlichster Natur, aus unterschiedlichsten Gründen und Materialien erbauen.

Eine sehr spezielle Tür-Art stellen Notausgangstüren [13] in schnellbefahrenen Bahntunnel dar, an die die unterschiedlichsten Anforderungen gestellt werden - u.a.:

- dauerhafter Widerstand gegenüber den auftretenden aerodynamischen Belastungen

- dauerhafte Funktionsfähigkeit (Öffnen- und Schließbarkeit)

- Druck-/, Rauch-/, Staubdichtigkeit

- $\quad$ Brandwiderstand (z.B. ETK/90 min.)

- Öffnungskraft < 100 Newton

- $\quad$ simple, robuste Mechanik bzw. einfache Wartbarkeit

Nachdem in den nächsten Jahren weitere lange und schnellbefahrene Bahntunnel im Netz der ÖBB in Betrieb genommen werden (u.a. Koralm, Semmering, Brenner), ist es von großer Bedeutung ausreichend dimensionierte und für eine Lebensdauer von mind. 30 bis 50 Jahre ausgelegte Türen zu entwickeln. Die Türen müssen über ihre gesamte Lebensdauer tragsicher, gebrauchstauglich, ermüdungssicher und funktionsfähig sein.

\section{Aktueller Stand - die NEUEN ÖBB-Tunneltüren}

Anlässlich der Wiener Dynamik Tage 2016 wurde schon über diesen Themenkreis mit dem Titel - Erste Schritte zur Entwicklung von „neuen“ ÖBB-Tunneltüren - berichtet [8]. In diesem Beitrag wurde auf Belastungs-/ Bemessungsansätze, auf die Ermittlung von Eigenfrequenzen bzw. Eigenperioden und auf die Definition von Belastungszyklen für 1:1 Realversuche in einer Versuchsanstalt eingegangen.

In der Zwischenzeit konnten die nachstehend kurz angeführten weiteren Schritte von Seiten der ÖBB Projektleitung Koralmbahn 1 unternommen werden:

- Erstellung technischer Vertragsbedingungen bzw. EU-weite Veröffentlichung der Ausschreibung Tunneltüren KAB (mehrstufiges Verhandlungsverfahren inkl. „Last an Best Offer")

Vortrag STEINER / REITERER

Dynamik Tage Wien 2021 
- $\quad$ Beauftragung von TÜV AUSTRIA TVFA mit der Durchführung von Eignungsprüfungen (Dauerschwing-/ und Dauerfunktionsversuchen) im Maßstab 1:1 an Tunneltüren die in (Stahlbeton-)Prüfrahmen eingebaut sind

- Herstellung eines Testaufbaus der gesamten Türkonstruktion im Stahlbetonprüfrahmen und Herstellung der Lasteinleitungskonstruktion sowie Fixierung an die zu prüfende Tür. Durchführung von Vorversuchen an jeweils einer Schiebe-/ und Drehflügeltür

- Durchführung von Dauerschwingversuchen (Eignungsprüfung) zur Untersuchung des Ermüdungsverhaltens von je 2 Schiebe-/, Gelenkschiebe-/ und Drehflügeltüren je Bieter, mit einer Prüfkraft von bis zu $35 \mathrm{kN}$, einer Prüffrequenz von $5 \mathrm{~Hz}$ und einer Lastspielzahl von 1,5 Mio.

Die Prüfkraft wird „kraftgeregelt“ über einen servohydraulischen Zylinder generiert und über eine eigens für die Versuche entwickelte Lasteinleitungskonstruktion flächig auf das Türblatt aufgebracht. Durch die Verwendung eines servohydraulischen Zylinders für die Kraftanregung ist die Wahl einer Prüffrequenz mit $5 \mathrm{~Hz}$ möglich. Nur mit dieser Anregungsart werden die bei Zugein- und Zugausfahrten sich einstellenden Druckwellen mit deren innerhalb von 0,05 Sekunden plötzlich auftretenden „Stosswirkung“ realitätsnah abgebildet $[9,10]$. Die bei den Dauerschwingversuchen anzusetzenden Druck-/ Sogbelastungen von $+/-7,5 \mathrm{kN} / \mathrm{m}^{2}$ für die KAB ergeben im Fall einer Schiebetüre eine Prüfkraft von rd. +/- 35 kN und bei einer Drehflügeltüre von rd. +/- 18 kN.

Jeder Dauerschwingversuch wird in vordefinierten Abständen (insgesamt 6 mal innerhalb der 1,5 Mio. Lastspiele) unterbrochen, um während dieser Unterbrechungen in Summe 10.000 Öffnungs-/ und Schließvorgänge (Dauerfunktionsprüfung inkl. Prüfung der TürSicherheitsfunktionen) durchzuführen. Damit wird die Funktionsfähigkeit der Tür über den gesamten Prüfablauf und somit über die geplante technische Lebensdauer von 30 Jahren überprüft.

Erst nach Abschluss aller Versuche und dem Vorliegen aller "positiven" Ergebnisse im Sinn den in der Ausschreibung definierten Anforderungen (keine Ermüdungsschäden + Nachweis der dauerhaften Funktionsfähigkeit), wird von Seiten der ÖBB-Infrastruktur AG die finale Vergabe der Tunneltüren für die KAB durchgeführt.

\section{Vortrag STEINER / REITERER}

Dynamik Tage Wien 2021 


\section{Belastungsaufbringung - Vergleich aktueller Methoden}

Bisher wurde bei diversen anderen europäischen Bahnprojekten mit einem ähnlichen Anforderungsprofil (z.B. Lötschberg, Gotthard Basistunnel $(\mathrm{CH})$ ) die Dauerlastprüfung an den Türen, die in einer Prüfkammer montiert wurden, durch über Hochleistungsventilatoren erzeugte Druck- und Sogstöße, durchgeführt. Es wurden dabei z.B. für den Fall Gotthard Basistunnel Versuche mit 500.000 Zyklen mit einer Zykluszeit von ca. 2 Sekunden (entspricht einer Prüffrequenz von $0,5 \mathrm{~Hz}$ ) am Institut für Fenstertechnik (ift) in Rosenheim (D) durchgeführt $[1 ; 2 ; 3 ; 4 ; 8]$. Im Fall der KAB würden die 500.000 Zyklen aber nur einer Nutzungsdauer der Türen von 15 Jahren entsprechend.

Eine vergleichbare Anlage (Dynos by Hodapp) steht der Fa. Hodapp (D) zur Verfügung, die Druckdifferenzen bis zu +/- 7,5 kN/m², eine max. Prüffläche von $2,4 \times 2,4 \mathrm{~m}$, eine Druckänderungsgeschwindigkeit bis zu $30 \mathrm{kPa} / \mathrm{sec}$ und Zykluszeiten von ca. 1,5 sec (entspricht einer theoretischen Prüffrequenz von $0,66 \mathrm{~Hz}$ für die Aufbringung der Druck- und Sogbelastungen) erzielen kann [12].

Diese Versuchsdurchführungen mit Druckluft sind einerseits mit einem relativ hohen technischen bzw. logistischen wie auch zeitlichen Aufwand verbunden. Ein Umstand, der sich natürlich auch bei den damit verbundenen Prüfkosten niederschlägt. Andererseits ist auch die Realitätsnähe der Versuche ein wesentlicher Punkt für den Nachweis der Tragsicherheit, Dauerhaftigkeit und Ermüdungssicherheit von Tunneltüren.

Mit den bei der Druckluftprüfung gewählten Prüffrequenzen im Bereich weit unter $1 \mathrm{~Hz}$ wird die reale aerodynamische Belastungssituation bei den Zugfahrten und hierbei insbesondere die Stosswirkung in keiner Weise nachgebildet. Wie vorstehend beschrieben und auch in der nachfolgenden Abbildung 4 dargestellt, kommt es bei der Zugeinfahrt in den Tunnel zu plötzlichen Drucksprüngen, die innerhalb einer Zeitdauer von nur 0,05 Sekunden auftreten. Wenn man die Zeitdauer dieses Drucksprunges zu einer „sinusförmigen“ Belastung ergänzt, dann kommt man nur dann auf die Nachbildung der Realität in den Dauerschwingversuchen, wenn die Prüffrequenz mit mindestens $5 \mathrm{~Hz}$ gewählt wird (Periode $\mathrm{T}=4 \cdot 0,05 \mathrm{~s}=0,20 \mathrm{~s} \rightarrow$ Frequenz $=1 / T=5 \mathrm{~Hz}$ ). Eine zu geringe Prüffrequenz bildet die Druckstöße nur unzureichend ab und somit wird die Tunneltüre auch nicht realitätsnah belastet. Die Materialermüdung und Effekte der Gestaltänderung von sicherheitsrelevanten Bauteilen der Tür (z.B. der Verankerungsriegel bei der Drehflügeltüre) sind sehr stark von der Belastungsdauer und somit von der Prüffrequenz abhängig.

\section{Vortrag STEINER / REITERER}

Dynamik Tage Wien 2021 


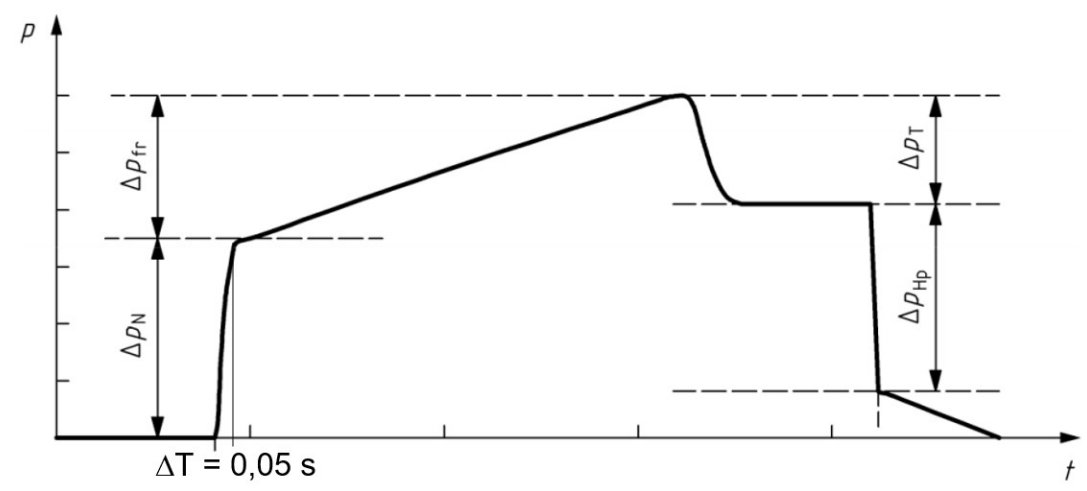

Abb 4: Allgemeine Darstellung der Drucksignatur eines Zuges bei der Einfahrt in einen Eisenbahntunnel [14]

Die Prüfung mit Druckluft stellt somit nur eine quasistatische Überprüfung der Dichtigkeit der Tunneltüren, jedoch aufgrund der fehlenden plötzlichen Lastaufbringung keine realitätsnahe Prüfung der Tragsicherheit, Ermüdungssicherheit und Funktionsfähigkeit der Tunneltüren dar. Durch Druck-/Sogmessungen in diversen Bestandstunnel im ÖBB-Netz, konnte eindeutig nachgewiesen werden, dass der Drucksprung bei der Zugeinfahrt in einem Bruchteil von einer Sekunde auftritt (siehe Abb. 5 / Portaleinfahrt Zugkopf).

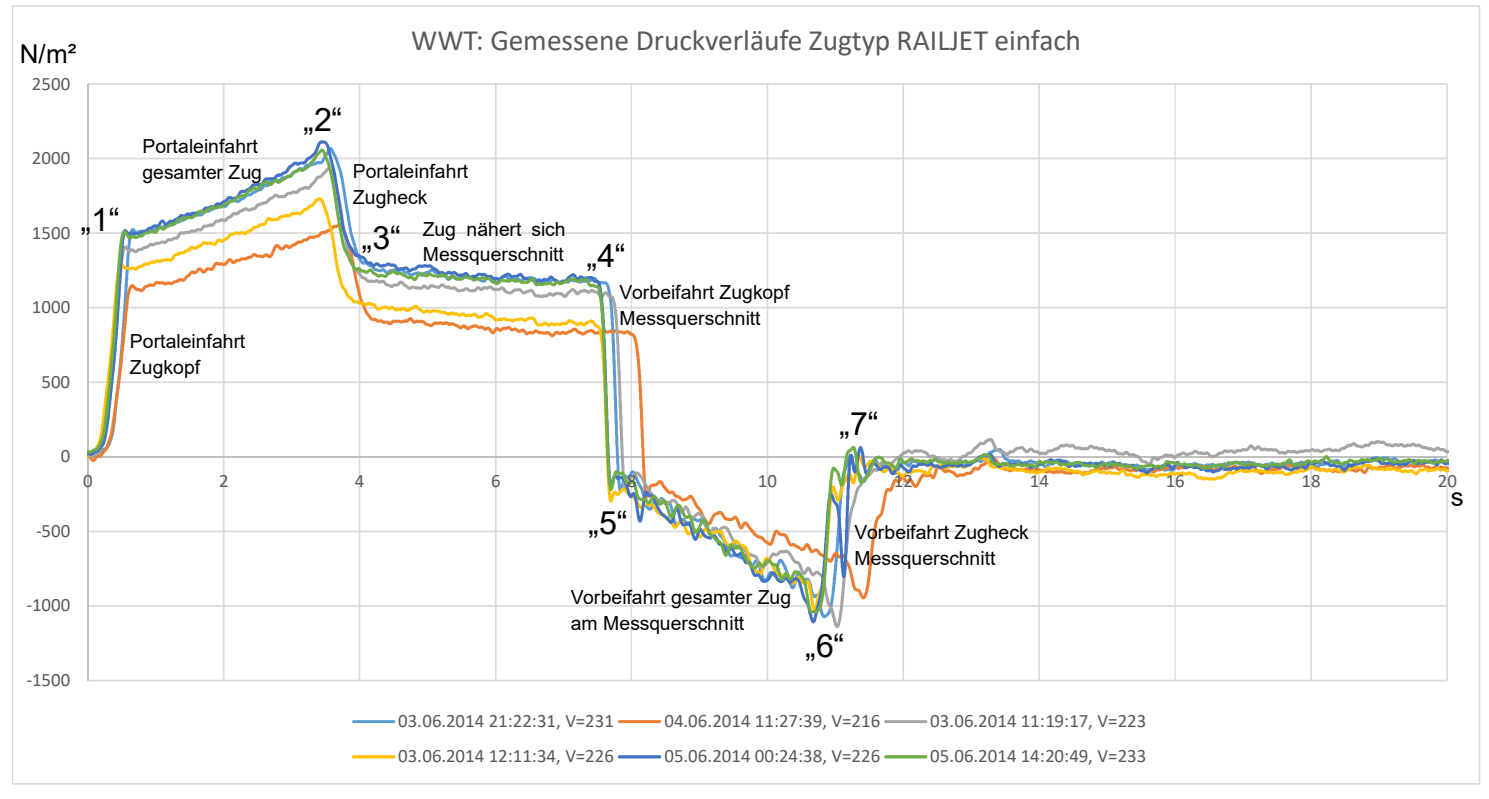

Abb. 5: Druckmessung Weststrecke / Wienerwaldtunnel [Revotec]

\section{Vortrag STEINER / REITERER}

Dynamik Tage Wien 2021 
Aus den angeführten Gründen wurde für die Tunnelprojekte der KAB entschieden, einen Prüfstand mit einem servohydraulischen Zylinder, einer Prüffrequenz von $5 \mathrm{~Hz}$ und einer Anzahl von 1,5 Mio. Lastwechsel für den Nachweis der Dauerhaftigkeit und Funktionsfähigkeit der Tunneltüren auszuführen.

Im Zusammenhang mit den vorstehend auch kurz angeführten bzw. in Verwendung stehenden "Luftdruck-Prüfständen", ergibt sich bei Anwendung der servohydraulischen Prüfmethode ein um einen Faktor von ca. 10 (0,5:5 Hz) geringerer Zeitaufwand für die Prüfung der Türen.

- $\quad$ Luftdruck-Prüfstand

1,5 Mio. Lastwechsel $\times 2$ Sekunden $=3$ Mio. Sec. $=$ rd. 830 Std. $=$ rd. 35 Tage

- Hydraulik-Prüfstand

1,5 Mio. Lastwechsel $\times$ 0,20 Sekunden $=300.000$ Sec. $=83$ Std. $=$ rd. 3,5 Tage

In Kombination mit der möglichen Anzahl der zu prüfenden Tunneltüren im Zuge eines Zulassungs-/ Vergabeverfahrens entsteht - nur unter Berücksichtigung der reinen Prüfzeiten - der nachstehend beschriebene Zeitunterschied.

Wenn z.B. je Bieter 2 Türen vom Typ Drehflügeltür (DFT), Schiebetür (ST) und Gelenkschiebetür (GST) geprüft werden, ergeben sich in Summe je Hersteller 6 Testtüren. Im Fall einer Luftdruckprüfung ergeben sich somit -35 Tage $\times 6$ Türen $=210$ Tage oder im anderen Fall $-3,5$ Tage x 6 Türen $=21$ Tage. D.h. einen Zeitunterschied von rd. 6 Monaten.

Unter Berücksichtigung weiterer Fixzeiten - u.a. der Montage der Tür im Prüfrahmen, der Installation der Belastungs-/ bzw. Messeinrichtung, Durchführung von Dauerfunktionsprüfungen (Öffnen- und Schließbarkeit) - kann im Fall 1 (Druckluftprüfung) von fast einem Jahr und im Fall der Prüfung mit servohydraulischen Zylinder von 3 bis 4 Monaten GesamtPrüfdauer ausgegangen werden.

Sind mehrere Bieter im Ablauf zu berücksichtigen, dann kann davon ausgegangen werden das sogar mehrere Jahre bis zu einem realen Vertragsabschluss vergehen können.

\section{Lasteinleitung bzw. Lastverteilung / servohydraulischer Zylinder - Türblatt}

Über eine entsprechende konstruktive Ausführung der Lasteinleitungskonstruktion (gelenkige Lagerungen der Stahlträger), konnte erreicht werden, dass die vom servohydraulischen Zylinder auf das Türblatt aufgebrachten Druck-/ wie auch Zugkräfte weitgehend zwängungsfrei und damit gleichmäßig verteilt eingetragen werden (s. Abb. 6). Im ersten Schritt wurden

Vortrag STEINER / REITERER

Dynamik Tage Wien 2021 
kreisrunde Lasteinleitungsplatten $(\varnothing 300 \mathrm{~mm}$ ) mit einem dauerhaft ablösesicheren doppelseitigen Klebeband auf der belasteten Seite der Türblattoberfläche aufgeklebt. Über Vorversuche wurde die Dauerhaftigkeit des verwendeten Klebebandes bei der dynamischen Zugbelastung von ca. $0,32 \mathrm{~N} / \mathrm{mm}^{2}$ positiv nachgewiesen. Im nächsten Schritt wurde die als statisch bestimmtes System ausgeführt Lasteinleitungskonstruktion über eine Schraubverbindung an die kreisrunden Lasteinleitungsplatten angeschlossen. Der servohydraulische Zylinder ist permanent mit der Lasteinleitungskonstruktion verschraubt und über einen Flaschenzug wird das Eigengewicht der gesamten Konstruktion zur Vermeidung einer zusätzlichen, in der Realität nicht auftretenden Belastung, kompensiert.
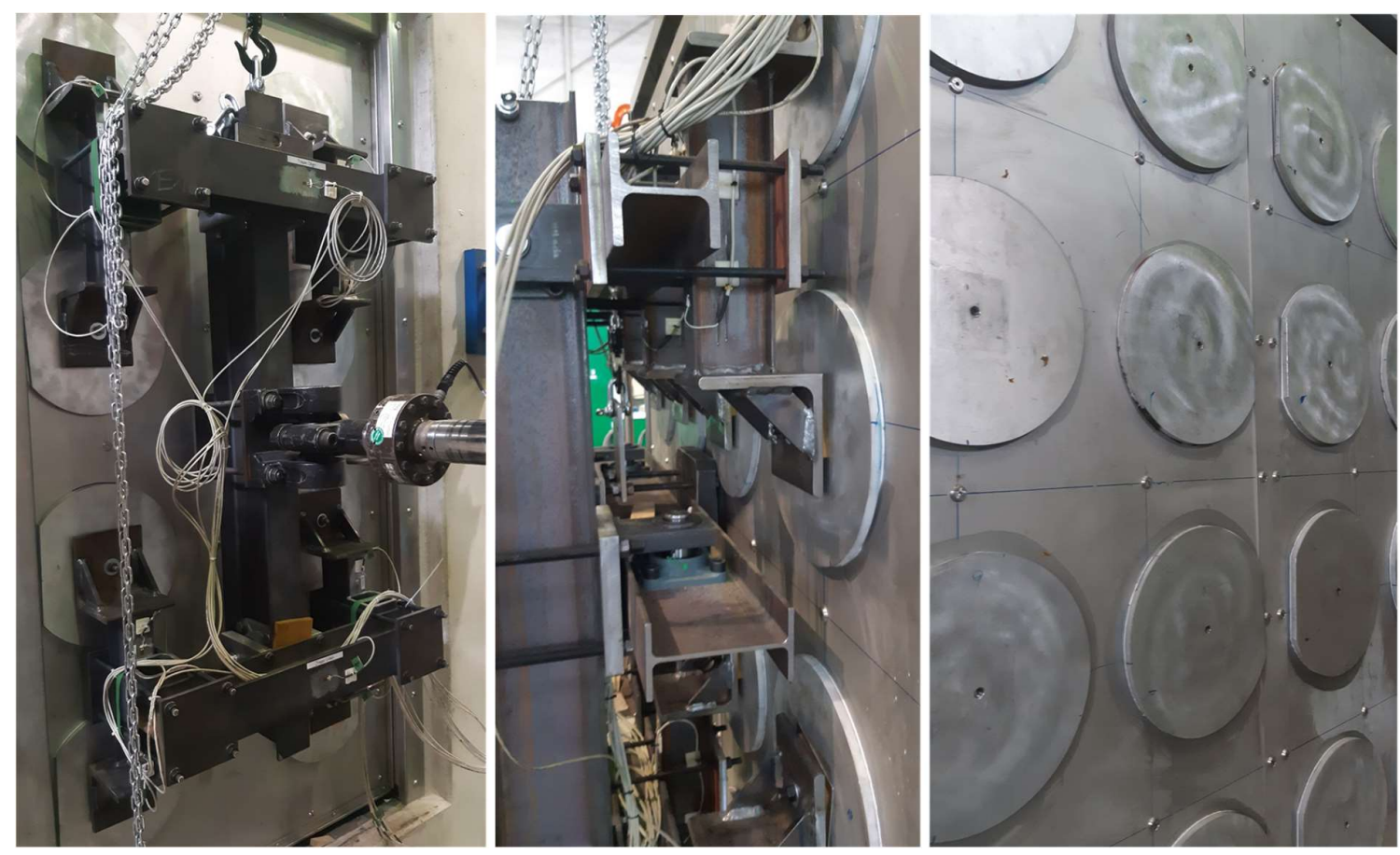

Abb. 6: Lastverteil-/ und Lasteinleitungskonstruktion zur Übertragung von Druck-/ Zugkräften - u.a. mit Aluminiumplatten und doppelseitigem Klebeband [ÖBB / Revotec / TÜV Austria TVFA]

Die möglichst gleichmäßige Verteilung der durch den servohydraulischen Zylinder erzeugten Einzelkraft über die Lasteinleitungskonstruktion in die einzelnen Lasteinleitungsplatten bzw. schlussendlich auf das Türblatt ist bei dieser Versuchsart von großer Wichtigkeit und daher wurden im Vorfeld umfangreiche numerische Untersuchungen zur Lastverteilung in einem

\section{Vortrag STEINER / REITERER}

Dynamik Tage Wien 2021 
Rechenmodell durchgeführt. Um diese „theoretischen“ Ansätze auch messtechnisch nachweisen zu können, wurde TÜV Austria TVFA beauftragt ein spezielles 3D Kamerasystem (ARAMIS / GOM GmbH a ZEISS company) einzusetzen, dass es ermöglicht 3-dimensionale Verformungen (Verschiebungen / Dehnungen) während unterschiedlicher vom servohydraulischen Zylinder erzeugten Belastungen (Druck / Zug) vollflächig und berührungslos festzuhalten.

Aus den beispielhaft in der Abb. 7 illustrierten Aufnahmen kann sehr gut erkannt werden, dass die entwickelte Konstruktion eine sehr ausgewogene Lastverteilung und damit einhergehende Verformung des gesamten Türblattes bewirkt. Die gleichmäßige Verteilung der Prüfkraft konnte damit positiv nachgewiesen werden.

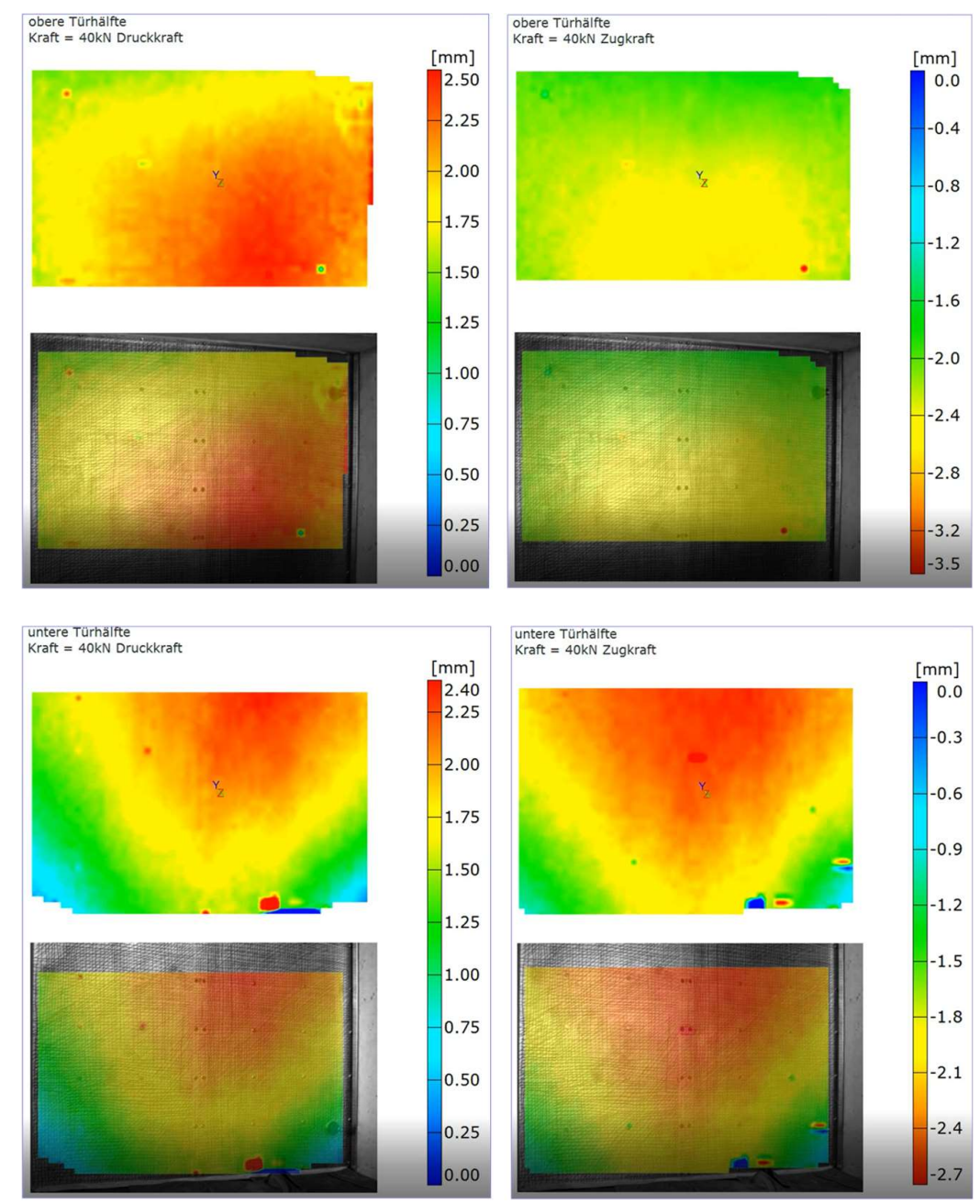

Abb. 7: Verformungen der oberen bzw. unteren Türhälfte bei 40 kN Druck- und Zugbelastung [TÜV Süd TVFA]

Vortrag STEINER / REITERER

Dynamik Tage Wien 2021 


\section{Zerstörungsfreie Zustandsprüfung der Tunneltür mittels periodischen Eigenfrequenzmessungen}

Wie schon im Beitrag 2016 [9] aufgezeigt, stellt die periodische Messung der Eigenfrequenz der Tunneltüre eine zerstörungsfreie Möglichkeit zur Ermittlung des aktuellen Zustandes der Türe dar. Die Türen wurden daher vor Versuchsbeginn, d.h. im Neuzustand und nach Aufbringen der 1,5 Millionen Belastungszyklen durch Einsatz eines elektrodynamischen Schwingerregers auf ihr dynamisches Antwortverhalten getestet. Das Ziel der Messungen war es, einen Vergleich der gemessenen Eigenfrequenzen vor Versuchsbeginn und nach Versuchsende durchzuführen und damit eine Aussage zum aktuellen Türzustand bzw. Rückschlüsse auf etwaige, während des Dauerschwingversuches aufgetretenen Zustandsveränderungen vornehmen zu können.

Die dynamischen Messungen wurden durch Anwendung eines sogenannten „Long Stroke Vibration Exciters“ krafterregt durchgeführt. Die Erregerfrequenz wurde im Bereich von 1 bis $100 \mathrm{~Hz}$ variiert und mit einem sogenannten linearen "Sinus-Sweep“ abgefahren. Die eingetragene Erregerkraft wurde über die gesamte Versuchsdauer mit einem Kraftsensor und die Schwingungsantwort der Tür mit Beschleunigungssensoren gemessen. Aus den Beschleunigungssensoren wurden die Zeitverläufe der Beschleunigungen, die Türeigenfrequenzen und die Schwingungsformen ermittelt sowie Performance-Indikatoren als geeignete Beurteilungsgrößen des Türzustandes abgeleitet. Zur Analyse des Frequenz-

änderungsverhaltens der Probetüren wurden die aufgezeichneten Zeitsignale mittels FFT in den Frequenzbereich transformiert.

Abb. 8 zeigt ein Foto von der Versuchsdurchführung und ein exemplarisches Ergebnis für das messtechnisch ermittelte Frequenzspektrum einer ST vor Versuchsbeginn (graue Linie) und nach Versuchsende (blaue Linie). Man erkennt, dass es in diesem Fall nach Aufbringen der 1,5 Millionen Lastzyklen zu signifikanten Eigenfrequenzverschiebungen gekommen ist. Die „Starrkörpereigenfrequenz" $f_{0}$ bildet sich nach Abschluss des Versuches nur noch ganz gering aus.

Dies wird auf Zwängungs-/ Verspannungseffekte bei Versuchsende zurückgeführt, nachdem derselbe Effekt bei bewusstem Auskeilen der Türe, d.h. bei Behinderung der Bewegungsmöglichkeit des Türflügels, beobachtet werden konnte. Bei den Eigenfrequenzen $f_{1}$ und $f_{2}$ kommt es ebenfalls zu Verschiebungen, wobei insbesondere die Eigenfrequenz $f_{2}$ eine große Abweichung zur Messung vor Versuchsbeginn aufweist.

\section{Vortrag STEINER / REITERER}

Dynamik Tage Wien 2021 
Eine Detailanalyse der aufgezeichneten Messdaten inklusive eines Versuches der Zuordnung zum Zustand der geprüften Türe wird derzeit durchgeführt.
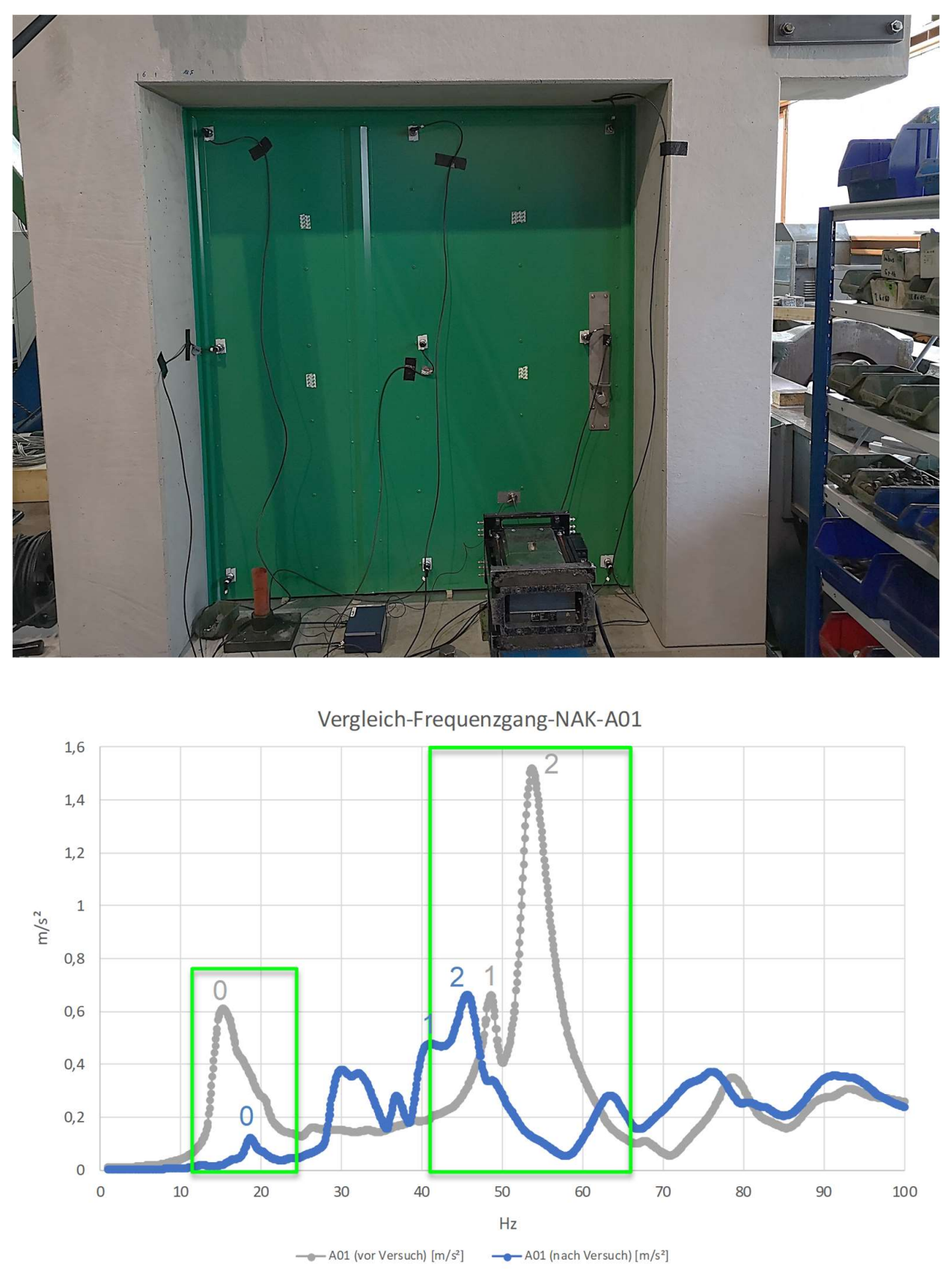

Abb. 8: Oben - Versuchsdurchführung mittels elektrodynamischen Schwingerreger Unten - Frequenzspektrum vor Versuchsbeginn (grau Linie) und nach Versuchsende (blaue Linie) [Revotec]

\section{Vortrag STEINER / REITERER}

Dynamik Tage Wien 2021 
Durch Anwendung der hier beschriebenen periodischen Eigenfrequenzmessungen an den real eingebauten Tunneltüren in diversen Tunnelbauten in Österreich könnte zerstörungsfrei auf ihren aktuellen Zustand rückgeschlossen und damit mögliche Austauschzyklen im Sinne einer vorausschauenden „prädiktiven Instandhaltung / predictive Maintenance“ genauer eingegrenzt werden. Dies würde für die ÖBB-Infrastruktur einen großen Vorteil in der Erhaltung darstellen.

\section{Zusammenfassung}

Aufbauend auf dem Wissensstand 2016 [9] wurde eine konsequente Weiterbearbeitung aller schon damals bekannten (u.a. Definition der einwirkenden Kräfte, Anzahl der Belastungszyklen) bzw. zwischenzeitlich neu aufgetretenen Gesichtspunkte und auch Problemen (u.a. Umsetzung einer praktikablen Prüfeinrichtung, Verifizierung von getätigten Annahmen, Evaluierung von erhaltenen Ergebnissen) betrieben. Ziel ist es mit Ende 2020 die Eignungsprüfungen mit allen Bietern abzuschließen und darauf aufbauend Anfang 2021 eine konkrete Vergabe tätigen zu können. Mit diesem geplanten weiteren Zeitablauf sollte gewährleistet sein, dass alle Einbaustellen entlang der KAB Graz - Klagenfurt (von Ost nach West - Flughafentunnel Graz (FTG), Koralmtunnel (KAT), Granitztaltunnel (GTT), Steintunnel (STT)) rechtzeitig die benötigten Tunneltüren in der entsprechenden Qualität für den Einbau zur Verfügung stehen.

\section{Literaturangaben}

[1] Berner Fachhochschule; Spezialisten für Sicherheit: HSB und HTI prüfen Tunneltüren für die Alp Transit Gotthard AG; Biel; Mai 2006

[2] Fabbri Davide, Strass Christian, Peggs Simon; Safety doors in world's longest tunnel under the Gotthard: Outstanding requirements and performance. Feedback from the bidding phase and results from the testing phase on selected prototypes; Proc. of the 33rd ITA AITES World Tunnel Congress; Prague; 5.-10. May 2007

[3] Haldimann Ruedi, Nyfeler Samuel, Reinke Peter; Mechanical equipment in long, highspeed rail tunnels - Specification and operational experience of doors, cabinets, containers and other elements in the Loetschberg Base Line; Proc. of the 33rd ITA AITES World Tunnel Congress; Prague; 5.-10. May 2007

[4] Peggs Simon, Pochop Frank; Anforderungen und ganzheitliche Projektabwicklung am Beispiel Gotthard-Basistunnel; Rosenheimer Tür- und Tortage 2008

[5] bmvit; The Baltic Adriatic Axis, Element of the future European TEN-T Core Network; 2010

[6] ÖBB-Infrastruktur AG; The Koralm Railway, A part of the new Southern Railway Line; 2012

\section{Vortrag STEINER / REITERER}

Dynamik Tage Wien 2021 
[7] Reiterer, M., Kari, H.; Generalized shock spectrum of structures in tunnels due to aerodynamic loading; Proceedings 13th International Railway Engineering Conference 2015, 30th June - 1st July 2015; Edinburgh, Scotland, UK

[8] Gotthard-Tunnel mit ift-geprüften Fluchttüren; Presseinformation 16-06-61 vom 8. Juli 2016; ift Homepage

[9] Steiner Helmut, Hannes Kari, Michael Reiterer; Erste Schritte zur Entwicklung von "neuen" ÖBB-Tunneltüren; Dynamik Tage Wien 2016

[10] Steiner Helmut, Hannes Kari, Michael Reiterer; Baudynamische Analysen bei der Entwicklung von Tunneltüren für die ÖBB: Simulationsberechnungen der Druck- und Sogbelastungen, Stoßspektren, Eigenfrequenzen, Ermüdungsbemessung; STUVA Conference 2017; Forschung + Praxis 49

[11] CEF support to Baltic-Adriatic Corridor; February 2018; European Commission, Brussels, Belgium

[12] Druck-/Sogprüfstand Fa. Hodapp; Vorstellung des Prüfstandes - Einladung 14. Mai 2019

[13] Wolfgang Baltzer, Torsten Brungsberg, Werner Riepe; Anforderungen an die Ausbildung von Notausgangstüren in Straßentunneln: Barrierefreiheit, Druck/Sogbelastungen, Geometrie, Ausführungsbeispiele; STUVA Conference 2019; Forschung + Praxis 53

[14] ÖNORM EN 14-067-5: Bahnanwendungen - Aerodynamik, Teil 5: Anforderungen und Prüfverfahren für Aerodynamik im Tunnel. Austrian Standard Institute, Ausgabe: 201102-01

Autorenkontakt: helmut.steiner2@oebb.at

michael.reiterer@revotec.at

Vortrag STEINER / REITERER

Dynamik Tage Wien 2021 